\title{
Clinical Surgery Made Easy: A Companion to Problem-Based Learning, Mohan De Silva
}

\author{
tfm Publishing, UK, 2008, 248 pp, 1st edn, \$90.00, ISBN 978-1-903378-65-6, paperback \\ (amazon.com)
}

\author{
Samir Johna
}

Published online: 14 July 2009

(C) Société Internationale de Chirurgie 2009

Reviewing medical or surgical textbooks is not necessarily my favorite intellectual activity given that many textbooks are stuffed with material that has no clinical application. Hence, they are somewhat boring to read. I wonder why psychologists and psychiatrists never thought of using them for hypnosis. As such when asked to review one, I commission my wife to ring a bell every 15 minutes to make sure that I stay on course until the job is done.

This book under review is clearly an exception. After studying the entire book, I find it an easy read and full of surgical gems. Also, it addresses a common obstacle in surgical training known to all surgical educators. Medical students and junior residents are often challenged by formulating a working diagnosis and a treatment plan for a wide range of surgical problems they face day in and day out throughout their training. Even in the presence of adequate knowledge, it is often difficult for them to apply it when required. Such skills can only be mastered through problem-based curricula and through the instruction of competent and dedicated clinical teachers, which, depending on the time of exposure and or the case load, are not necessarily available to all trainees at all times. To circumvent this situation, Professor De Silva carefully designed a series of common clinical scenarios in general surgery to stimulate the clinical decision-making approach. Under each topic he highlighted the essential core knowledge and the relevant clinical pathways, followed by questions and answers. This reinforces retention and facilitates recall of facts when required.

S. Johna $(\bowtie)$

Loma Linda University School of Medicine, Loma Linda, CA,

USA

e-mail: samir.johna@gmail.com
On flipping through the pages of this book I get the sense that it is written by a skilled, experienced clinical surgeon who has mastered both the art and science of surgery. Some of the chapters are extremely well written, particularly those dealing with the gastrointestinal tract and the thyroid gland. As such, promoters of this book are right on the money when recommending it to medical students, residents, and may be junior surgeons. However, I believe it is an invaluable asset for the seasoned surgeon as well, particularly when engaged in teaching. As we all know, there are gaps at the level of thinking between the teaching faculty and their students and trainees. This book bridges such gaps and provides a framework for all involved parties to stay on the same page in a focused, well controlled manner. It ensures the best of learning particularly when there is limited time of exposure.

As to potential deficiencies, if present they are minor and would not diminish the value of this valuable work. Some topics would have been more appropriate if started at an earlier stage, taking the reader through the indicated workup as patients do not present as surgical versus medical cases but, rather, with a sign and or a symptom. For example, abdominal pain would have been a better topic than separating the pain into biliary colic, acute pancreatitis, or acute abdomen; groin mass or groin pain would have been a better topic than groin hernia. The same goes for topics on abdominal mass, goiter, and hypercalcemia instead of the current respective topics.

After all is said and done, the author has successfully assembled an amazing array of facts and has woven them into a fabric fit for trainees and educators alike. 